\title{
FRECUENCIA DE PRESENTACIÓN DE INESTABILIDAD LUMBOSACRA EN CANINOS LABRADOR RETRIEVER
}

\author{
Frequency of Presentation of Lumbosacral Instability in Labrador \\ RETRIEVER DOGS
}

\author{
Dennis Arana C. ${ }^{1,3}$, Diego Díaz C. ${ }^{1,4}$, Víctor Fernández A. ${ }^{1}$, César Gavidia C. ${ }^{2}$, \\ Vicente Chilón C. ${ }^{1}$
}

\section{Resumen}

El objetivo del estudio fue determinar la frecuencia de presentación de la inestabilidad lumbosacra en caninos Labrador Retriever mayores de seis meses de edad, así como su posible asociación con las variables sexo, edad, peso, signos radiológicos y el signo clínico de dolor lumbosacro. El estudio se realizó mediante la evaluación radiográfica de 60 caninos escogidos al azar y distribuidos según sexo (30 por sexo), edad (3 grupos etarios) y peso (3 categorías). La frecuencia de inestabilidad lumbosacra fue $75.0 \%$ (45/ $60)$. Las hembras fueron las más afectadas $(86.7 \%, 26 / 30)$ y la proporción macho:hembra fue de 1:1.4. No se obtuvo una asociación estadística con la edad. Los animales obesos (sobrepeso $>15 \%$ sobre el límite estándar) mostraron el mayor porcentaje de presentación $(87.5 \%, 21 / 24)$. El signo radiológico más frecuente fue la subluxación ventral del sacro en relación a $\mathrm{L}_{7}(75.0 \%, 45 / 60)$. La palpación transrectal permitió detectar la mayor frecuencia de dolor lumbosacro $(80.0 \%, 45 / 60)$. La evaluación radiológica de la enfermedad mostró un grado de concordancia muy alto con la evaluación del dolor lumbosacro $(\mathrm{p}<0.05)$.

Palabras clave: inestabilidad lumbosacra, caninos, Labrador Retriever, evaluación radiológica

\section{Abstract}

The aim of the study was to determine the frequency of lumbosacral instability in Labrador Retriever dogs older than six months of age, and to determine its association with the variables sex, age, body weight, radiological signs, and clinical signs of lumbosacral pain. The disease was detected by radiographic evaluation. Sixty dogs were randomly selected and distributed according to sex (30 per sex), age (3 groups) and body weight (3 categories). The frequency of lumbosacral instability was $75.0 \%(45 / 60)$. The

\footnotetext{
${ }^{1}$ Clínica de Animales Menores, ${ }^{2}$ Laboratorio de Medicina Veterinaria Preventiva, Facultad de Medicina Veterinaria, Universidad Nacional Mayor de San Marcos, Lima

${ }^{3}$ E-mail: dennis_arana_vet@yahoo.es

${ }^{4}$ E-mail: holadiegoperu@hotmail.com
} 
females were most affected $(86.7 \%, 26 / 30)$ and male:female ratio was 1:1.4. None statistical association was found due to age. Obese animals ( $>15 \%$ overweight on the limit of the standard) showed greater frequency $(87.5 \%, 21 / 24)$. The most frequent radiological sign was the ventral subluxation of the sacrum relative to $\mathrm{L}_{7}(75.0 \%, 45 / 60)$. Transrectal palpation allowed the highest detection of lumbosacral pain $(80.0 \%, 45 / 60)$. The radiological evaluation of the disease showed a high degree of concordance with the evaluation of the lumbosacral pain $(\mathrm{p}<0.05)$.

Key words: lumbosacral instability, canine, Labrador Retriever, radiological evaluation

\section{INTRODUCCIÓN}

En la especie canina existen diversas alteraciones que afectan la columna vertebral. Uno de los segmentos afectados es el segmento lumbosacro, donde se encuentra la cauda equina, conjunto de nervios periféricos que abandonan caudalmente la médula espinal (Suwankong et al., 2008).

La articulación lumbosacra es el punto de transferencia de la mayor parte de las fuerzas, viéndose sometida a flexiones y rotaciones anómalas que pueden llegar a causar procesos degenerativos, pese a los fuertes ligamentos que la sustentan. Una de las afecciones más frecuentes a este nivel es la inestabilidad lumbosacra (ILS), donde se comprimen las estructuras nerviosas y la articulación se vuelve más sensible a las fuerzas mecánicas (Bojrab, 2001; Barrios et al., 2008). Esta patología afecta principalmente a razas grandes como Pastor Alemán, Gran Danés, Airedale Terrier, Setter Irlandés, Springer Spaniel Inglés, Labrador Retriever, Golden Retriever y Boxer, entre otros. Se presenta en un rango amplio de edades (2-13 años), aunque es más común entre los 6 y 7 años de edad, y con mayor frecuencia en machos (Santoscoy, 2006).

En el canino, los signos clínicos de las lesiones lumbosacras difieren de los observados en otras zonas de la columna vertebral, dado que a este nivel no se presenta la médula espinal como tal, sino tan sólo la cauda equina. Sin embargo, es factible que la en- fermedad de la columna lumbar baja tenga un efecto importante, ya que puede comprometer, en forma individual o en conjunto, varios nervios que controlan la locomoción, la continencia fecal y urinaria, y la sensibilidad de los miembros posteriores (Suwankong et al., 2008).

Los mecanismos responsables de la ILS no están claros, pero la respuesta a esta patología, como un mecanismo del cuerpo por compensar o aumentar la estabilidad, es el inicio de cambios proliferativos en distintas estructuras, tales como esclerosis de la placa terminal lumbosacra, osteofitos de las facetas articulares, hipertrofia del ligamento interarcual y de la cápsula articular de las facetas, y protrusión del anillo dorsal (Santoscoy, 2006; Barrios et al., 2008).

El presente estudio tuvo por objetivo diagnosticar clínica y radiológicamente y determinar la frecuencia de presentación de la inestabilidad lumbosacra en la raza Labrador Retriever, así como establecer su asociación con las variables sexo, edad, peso, signos radiológicos y signos clínicos.

\section{Materiales y Métodos}

\section{Lugar de Estudio y Animales}

El presente estudio se realizó en la Clínica de Animales Menores de la Facultad de Medicina Veterinaria, Universidad Nacional Mayor de San Marcos, Lima. 
El tamaño muestral correspondió a 60 canes (30 por sexo) Labrador Retriever, mayores de seis meses de edad, que llegaron a la clínica por varios motivos de atención (consultas, tratamientos, ecografías, radiografías, baños, etc.), y que fueron incluidos en el estudio con la autorización de sus propietarios. Los canes fueron distribuidos en tres grupos etarios $(\mathrm{GE} 1=0.5$ a 3.9 ; $\mathrm{GE} 2=4.0$ a 7.9 ; $\mathrm{GE} 3=8.0$ a más años de edad) y tres categorías de peso vivo $[\mathrm{CP} 1=$ peso estándar de la raza (machos: 27-36 kg; hembras: 25-32 $\mathrm{kg}$ ); $\mathrm{CP} 2=$ sobrepeso (hasta el $15 \%$ sobre el límite superior del peso estándar); $\mathrm{CP} 3=$ obesidad (mayor al 15\% sobre el límite superior del peso estándar)].

\section{Procedimientos}

Los animales fueron sometidos a una evaluación subjetiva del dolor lumbosacro, donde se consideró que el animal presentaba dolor cuando manifestaba temblores musculares de los miembros posteriores, vocalizaba o intentaba girar para morder.

Se utilizaron cuatro métodos de evaluación (con el animal en estación):

a) La presión digital dorsal de la articulación lumbosacra, presionando hacia ventral con uno o ambos dedos pulgares en el punto medio entre las dos alas del íleon (teniéndolas como base de apoyo);

b) La prueba de lordosis, que se realizó con presión digital dorsal pero con la extensión hacia caudal de uno o ambos miembros posteriores;

c) La extensión y tracción de la cola, tomando la cola de manera extendida y llevándola hacia craneal y por encima de la grupa siguiendo la línea de la columna vertebral;

d) La palpación transrectal de la articulación lumbosacra, presionando hacia dorsal la cara ventral de la articulación lumbosacra, a través del recto.

Se hicieron tomas radiográficas con un equipo móvil de Rayos X General Electric ${ }^{\circledR}$ modelo Mobile 225 de columna móvil, con capacidad máxima de $125 \mathrm{kVp}$ y de $200 \mathrm{~mA}$ y películas radiográficas Kodak ${ }^{\circledR}$ (Medical XRay Film General Purpose Green - MXG 27.9 x $35.6 \mathrm{~cm}$ ). Para la toma de las placas radiográficas, los animales fueron sedados con $5 \mathrm{mg} / \mathrm{kg}$ de clorhidrato de ketamina al $10 \%$ y $0.1 \mathrm{mg} / \mathrm{kg}$ de maleato de acepromazina al $1 \%$, y fueron colocados en posición decúbito lateral con el área lumbosacra sobre el chasis de la película. La cabeza se mantuvo en posición relajada, los miembros anteriores se colocaron en paralelo con una ligera proyección hacia craneal y los miembros posteriores se colocaron de forma paralela y se sujetaron manteniendo una posición anatómicamente normal con respecto a la articulación lumbosacra. La técnica radiológica, el kilovoltio-potencia $(\mathrm{kVp})$ y los miliamperios $(\mathrm{mA})$ fueron regulados según el protocolo de exposición en pequeños animales (Connie y Cheryl, 2000).

En el estrechamiento del espacio intervertebral de la articulación lumbosacra, se evaluó la presencia o ausencia de aproximación de los cuerpos vertebrales $\mathrm{L}_{7}$ y $\mathrm{S}_{1}$; la radio-opacidad del agujero de conjunción de la articulación lumbosacra; y la presencia o ausencia de esclerosis de los bordes articulares de los cuerpos vertebrales de la articulación lumbosacra. En la espondilosis deformante se evaluó la presencia o ausencia de mineralización del ligamento intervertebral ventral. En los cambios en la forma de $\mathrm{L}_{7}$ se evaluó la presencia o ausencia de cambios morfológicos en el cuerpo vertebral; y en el desplazamiento ventral del sacro en relación a $\mathrm{L}_{7}$ se evaluó la presencia o ausencia de deslizamiento hacia ventral del sacro.

\section{Análisis Estadístico}

Se determinaron las frecuencias porcentuales de la ILS para signos radiológicos, signos clínicos de dolor lumbosacro, sexo, edad y peso. La asociación entre la presencia de signos radiológicos y la presencia del signo clínico de dolor lumbosacro con las va- 
Cuadro 1. Valores promedio y desviación estándar de edad (años) y peso (kg) según el sexo de canes Labrador Retriever

\begin{tabular}{lcccccc}
\hline \multirow{2}{*}{ Sexo } & \multicolumn{3}{c}{ Edad } & & \multicolumn{2}{c}{ Peso } \\
\cline { 2 - 3 } \cline { 6 - 7 } & Promedio & d.e. & & Promedio & d.e. \\
\hline Macho $(\mathrm{n}=30)$ & 5.6 & 3.4 & & 38.1 & 2.9 \\
Hembra $(\mathrm{n}=30)$ & 6.4 & 3.4 & & 36.7 & 4.1 \\
\hline Total $(\mathrm{n}=60)$ & 6.0 & 3.4 & & 37.4 & 3.6 \\
\hline
\end{tabular}

Cuadro 2. Frecuencia de canes Labrador Retriever positivos a inestabilidad lumbosacra con respecto a signos radiológicos y al dolor lumbosacro

\begin{tabular}{lccccc}
\hline \multirow{2}{*}{ Sexo } & \multicolumn{2}{c}{ Signos radiológicos } & & \multicolumn{2}{c}{ Dolor lumbosacro } \\
\cline { 2 - 3 } \cline { 6 - 6 } & $\mathrm{P}(+)$ & $\%$ & & $\mathrm{P}(+)$ & $\%$ \\
\hline Macho & $19 / 30$ & $63.3^{\mathrm{a}}$ & & $20 / 30$ & $66.7^{\mathrm{a}}$ \\
Hembra & $26 / 30$ & $86.7^{\mathrm{b}}$ & & $28 / 30$ & $93.3^{\mathrm{b}}$ \\
\hline Total & $45 / 60$ & 75.0 & & $48 / 60$ & 80.0 \\
\hline
\end{tabular}

${ }^{a, b}$ Superíndices diferentes dentro de columnas indican diferencia estadística $(p<0.05)$

riables sexo, edad y peso fue analizada mediante la prueba de Chi cuadrado con un nivel de significancia de 0.05 . La comparación entre los resultados obtenidos de la evaluación radiológica y de la evaluación clínica se realizó mediante la prueba de índice de Kappa.

\section{Resultados}

Los valores promedio de peso de los animales se muestran en el Cuadro 1. El Cuadro 2 muestra el porcentaje de animales positivos a ILS con respecto a los signos radiológicos y al signo clínico de dolor lumbosacro.
La mayor frecuencia de animales positivos a signos radiológicos $(80.0 \%, 16 / 20)$ se presentó tanto en canes entre 4.0 y 7.9 años como en aquellos con 8.0 a más años de edad. En machos, la mayor frecuencia $(72.7 \%, 8 /$ 11) se presentó entre 4.0 y 7.9 años y en hembras $(91.7 \%, 11 / 12)$ entre 8.0 a más años de edad. Asimismo, la mayor frecuencia de animales positivos al signo clínico de dolor lumbosacro fue de $85.0 \%$ (17/20), tanto en canes entre 4.0 y 7.9 años como en aquellos con 8.0 o más años de edad. En machos, la mayor frecuencia $(75.0 \%, 6 / 8)$ se encontró en canes con 8.0 o más años de edad, y en hembras $(100 \%, 9 / 9)$ entre 4.0 y 7.9 años de edad. No se encontró una asociación estadística entre la edad y los signos radiológicos o el signo clínico de dolor lumbosacro. 
La mayor frecuencia de animales positivos a signos radiológicos con respecto al peso $(87.5 \%, 21 / 24)$, se presentó en animales con un peso $>15 \%$ sobre el límite superior del peso estándar. No obstante, al estratificar por sexo, la mayor frecuencia en machos $(81.8 \%, 9 / 11)$ fue en grupo de peso estándar, y en hembras $(90.5 \%, 19 / 21)$ en el grupo con un peso $>15 \%$ sobre el límite superior del peso estándar $(\mathrm{p}<0.05)$. La mayor frecuencia de animales positivos al signo clínico de dolor lumbosacro fue $92.7 \%(22 / 24)$ para los animales con un peso $>15 \%$ sobre el límite superior del peso estándar, aunque no se encontró una asociación estadística entre la presentación del signo clínico de dolor lumbosacro y el peso.

Entre los signos radiológicos evaluados, el que presentó una mayor frecuencia fue el desplazamiento ventral (subluxación) del sacro en relación a $\mathrm{L}_{7}(75.0 \%, 45 / 60)$. Al comparar los resultados obtenidos de la evaluación radiológica y de la evaluación clínica para llegar al diagnóstico positivo de presentación de ILS, se encontró que ambos guardan un grado de concordancia muy alto $(k=0.86$, $\mathrm{p}<0.05$ ).

\section{Discusión}

Se halló una alta frecuencia de presentación de ILS en canes Labrador Retriever (75\%, 45/60) mediante la evaluación radiológica, frecuencia bastante similar a los datos obtenidos por Ness (1994) y Suwankong et al. (2008), quienes reportan frecuencias de 70.0 y $78.8 \%$, respectivamente, en estudios con varias razas, incluyendo el Labrador Retriever.

Se encontró una relación entre el sexo y la presentación de ILS, siendo las hembras las que presentaron una mayor frecuencia de la enfermedad, lo que se traduce en una proporción macho:hembra de 1:1.4. Los resultados difieren grandemente con otras publicaciones donde señalan que los machos son los más afectados, con proporciones que van desde 1.3:1 a 5:1 (Watt, 1991; Danielsson y Sjöström, 1999; Janssens et al., 2000).

No se encontró asociación estadística entre la presentación de ILS y la edad de los animales. En el presente estudio se consideró la edad de seis meses como edad mínima para el muestreo, debido a que se trata de una enfermedad muy ligada al desarrollo esquelético, razón por la cual los cambios iniciales o primeras manifestaciones de la misma deberían presentarse en la etapa de mayor crecimiento del individuo. Esto se confirma por el hallazgo de $3 / 5$ animales $(60 \%)$ menores de 2 años de edad positivos a la enfermedad; sin embargo, la edad no ha sido tomada como un factor de riesgo por otros autores, quienes tan sólo señalan un amplio rango de presentación que va desde los 2 hasta los 13 años con una media entre los 6 a 7 años (Indrieri, 1998; Danielsson y Sjöström, 1999; De Risio et al., 2000; Barrios et al., 2008).

Suwankong et al. (2008) halló que el desplazamiento ventral (subluxación) del sacro en relación a $\mathrm{L}_{7}$ fue el signo radiológico con mayor presentación en animales con ILS (78.8\%); similar al presente estudio donde esta característica se presentó en el $75 \%$ de los casos, constituyéndose en el signo radiológico más significativo para llegar al diagnóstico de ILS.

Por otra parte, en el estudio de Barrios et al. (2008), la evaluación clínica mostró que la prueba de lordosis era el principal método de evaluación del dolor a nivel de la articulación lumbosacra con un $90 \%$ de presentación, mientras que la presión digital y la palpación transrectal tenían una frecuencia de 50 y $20 \%$, respectivamente. Estos resultados difieren de los hallados en el presente estudio, donde la palpación transrectal fue el mejor método de evaluación, con un $80 \%$ de presentación, seguido de la tracción o extensión de la cola, prueba de lordosis y presión digital con 40,30 y $25 \%$, respectivamente. Tales 
diferencias pudieron deberse al carácter subjetivo de estas evaluaciones; además, se tiene que algunos animales requieren una evaluación más invasiva para poder demostrar el dolor en esa zona, y otros, a los cuales los leves cambios óseos aún no fueron capaces de comprimir la médula y, por lo tanto, no presentaban dolor a evaluaciones menos drásticas.

El alto grado de concordancia $(k=0.86$, $\mathrm{p}<0.05)$ entre la evaluación radiológica y la evaluación clínica que dieron resultados positivos a ILS muestra que ambas evaluaciones tienen similar valor diagnóstico, en contraste con lo citado por Scharf et al. (2004), quienes no hallaron una correlación entre los hallazgos clínico-neurológicos y los hallazgos radiológicos de su evaluación.

\section{Conclusiones}

- La frecuencia de presentación radiológica de ILS en la raza Labrador Retriever fue de $75.0 \%$. Las hembras fueron significativamente más afectadas $(86.7 \%, \mathrm{p}<0.05)$.

- No se encontró asociación entre la inestabilidad lumbosacra con la edad de los animales.

- El signo radiológico más frecuente para llegar al diagnóstico de la ILS fue el desplazamiento ventral (subluxación) del sacro en relación a $\mathrm{L}_{7}$.

- Se halló un grado de concordancia muy alto entre la evaluación radiológica y la evaluación clínica.

\section{Literatura Citada}

1. Barrios NS, Gómez M, Mieres M. 2008. Descripción de estenosis degenerativa lumbosacra mediante signos clínicos y tomografía computarizada en 10 perros adultos. [Internet], [20 no- viembre 2009]. Disponible en: http:// www.neurolatinvet.com/publi/ Trabajo\%20Estenosis\%20degenerativa\%20lumbosacra.pdf

2. Bojrab J. 2001. Técnicas actuales en cirugía en animales pequeños. $3^{\mathrm{a}}$ ed. Buenos Aires: Inter-Médica. 1276 p.

3. Connie M, Cheryl H. 2000. Diagnóstico práctico por imagen para técnicos veterinarios. Madrid: Acribia. 295 p.

4. Danielsson F, Sjöström L. 1999. Surgical treatment of degenerative lumbosacral stenosis in dog. Vet Surg 28: 91-98.

5. De Risio L, Thomas W, Sharp N. 2000. Degenerative lumbosacral stenosis. Vet Clin N Am-Small 30: 111129.

6. Indrieri RJ. 1988. Lumbosacral stenosis and injury of the cauda equina. Vet Clin N Am-Small 18: 697-709.

7. Janssens LAA, Moens Y, Coppens P, Peremans $K$, Vinck $H .2000$. Lumbosacral degenerative stenosis in the dog. Vet Comp Orthopaed 13: 97-103.

8. Ness MG. 1994. Degenerative lumbosacral stenosis in the dog: a review of 30 cases. J Small Anim Pract 35: 185190.

9. Santoscoy C. 2006. Estenosis vertebral lumbosacra (síndrome de cauda equina). AMMVEPE 17(2): 34-39.

10. Scharf $G$ Steffen $F$, Grünenfelder $F$, Morgan JP, Flückiger M. 2004. The lumbosacral junction in working German Shepherd dogs - Neurological and radiological evaluation. J Vet Med A 51: 27-32.

11. Suwankong N, Meij BP, Voorhout G, de Boer AH, Hazewinkel HA. 2008. Review and retrospective analysis of degenerative lumbosacral stenosis in 156 dogs treated by dorsal laminectomy. Vet Comp Orthop Traumatol 21: 285-293.

12. Watt PR. 1991. Degenerative lumbosacral stenosis in 18 dogs. J Small Anim Pract 32: 125-134. 